\title{
marges Marges
}

revue d'art contemporain Revue d'art contemporain

$08 \mid 2008$

L'art à l'heure de la société de services

\section{Un art « délivré »}

Sur quelques utilisations du service postal depuis les années 1960

A "Delivered" Art: About a Few Uses of the Postal Service Since the Sixties

\section{Marie Boivent}

\section{CpenEdition}

\section{Journals}

Édition électronique

URL : http://journals.openedition.org/marges/572

DOI : $10.4000 /$ marges. 572

ISSN : 2416-8742

Éditeur

Presses universitaires de Vincennes

\section{Édition imprimée}

Date de publication : 15 octobre 2008

Pagination : $51-69$

ISBN : 978-2-84292-252-8

ISSN : $1767-7114$

Référence électronique

Marie Boivent, « Un art « délivré » », Marges [En ligne], 08 | 2008, mis en ligne le 15 octobre 2009, consulté le 19 avril 2019. URL : http://journals.openedition.org/marges/572 ; DOI : 10.4000/ marges.572 


\section{Un art « délivré »:}

\section{Sur quelques utilisations du service postal depuis les années 1960}

L'art connaît au début des années 1960 de profonds changements, provoqués par une réaction critique, voire exaspérée, de nombreux artistes qui, depuis plusieurs années, se sentent en profond décalage avec ce qu'il est devenu. Face à la marchandisation de l'art et de ses " objets ", devenus à proprement parler des biens de consommation (qui plus est, réservés à une "élite »), mais également face au peu de place laissé à un art qui ne répond pas aux règles déterminées par les institutions en place - et par les préceptes greenbergiens -, les artistes cherchent des moyens pour répliquer. Réagir consistera notamment à trouver des espaces alternatifs où la sélection sévère, voire la censure, des tenants des lieux officiels de monstration de l'art ne sont plus à craindre. Les stratégies qui se mettent alors en place, liées à une volonté quelque peu utopique de rendre l'art accessible au plus grand nombre, vont aussi se révéler être de véritables remises en cause du mode « légitime » d'exposition d'une production artistique. Court-circuiter cette longue chaîne d'intermédiaires (galeries, marchands, musées mais aussi publicité) - symptomatique d'une société de service pour laquelle les modalités de diffusion et le label priment sur la valeur inhérente à l'objet - devient ainsi la priorité d'un certain nombre d'artistes. L'une des solutions envisagées, qui va s'incarner 
${ }^{1}$ Hervé Fischer rappelle que « les mass media ne diffusent qu'une part limitée de la production culturelle d'une société ", laissant ainsi en marge «ce qu'on appelle à tort ou à raison la culture de masse tout un champ d'expression jugé trop nouveau ou trop spécialisé par les médiateurs du grand public. » (Hervé Fischer, Art et communication marginale. Tampons d'artistes, Paris, Balland, 1974, p. 5).

${ }^{2}$ Grâce à la généralisation des photocopieurs dans les bureaux à partir des années 1960 par exemple. (ou tenter de s'incarner) sous différentes formes ou media, passe par l'idée d'une œuvre qui puisse rétablir un rapport plus direct au spectateur: par l'envoi postal notamment, elle peut désormais se « consulter » directement chez soi et n'est plus réservée à un acquéreur privilégié. Dès lors un paradoxe s'installe : pour échapper à un système d'échange fortement réglementé et reprendre leurs droits sur leur production, les artistes vont choisir de se confronter à d'autres ensembles de lois - tout aussi contraignants - attachés à d'autres institutions, en particulier celle qui régit la circulation par voie postale.

Cette étude se proposera de réexaminer cette tendance en rappelant ce qu'avaient à gagner les artistes à échanger un service contre un autre. Il s'agira dans un premier temps de voir quelles « formes » d'art sont susceptibles d'utiliser à des fins artistiques les services proposés par l'institution postale, du mail art - non pas considéré en tant que "concept d'école » mais par la « mise en circulation » qu'il provoque - aux publications d'artistes et particulièrement aux périodiques (et au principe d'abonnement qui leur est attaché).

Au-delà d'une simple appropriation d'un mode de communication spécifique et de ses règles inhérentes, il s'agira encore de voir quels sont les véritables enjeux de telles pratiques, tant du côté du « récepteur » que de celui du « créateur ». Car cet « art livré à domicile » - qui aujourd'hui peut nous apparaître comme un service par excellence - s'accompagne d'une réévaluation fondamentale de la conception de l'art, de sa diffusion et de sa réception. Ces pratiques qui ont connu un développement sans précédent au milieu des années 1960 et au début des années 1970, repensées et adaptées avec les moyens et habitudes d'aujourd'hui, restent d'une grande actualité par les alternatives qu'elles proposent.

\section{Les supports imprimés : un espace alternatif autonome}

Afin de se dégager définitivement de toute forme de «tutelle », il importait de trouver un medium ne nécessitant aucun intermédiaire, aucun compromis (aussi bien en ce qui concerne la "forme » que le «fond»), un medium ne dépendant d'aucune autorisation des détenteurs des réseaux de communication ${ }^{1}$ pour occuper le terrain. Cette nouvelle position - tant physique que politique - a su s'incarner avec force dans la forme imprimée, forme qui de plus a l'avantage de permettre à l'artiste d'assurer seul toutes les étapes de la fabrication ${ }^{2}$. En effet, si de nombreuses expériences visant à emprunter de nouveaux supports et canaux ont été tentées - telles 
les vidéos d'artistes câblo-diffusées -, peu ont offert aux artistes une liberté comparable à celle qu'ils ont trouvé avec le support imprimé.

Les commentateurs ont d'ailleurs su repérer le caractère éminemment alternatif des publications d'artistes, comme en témoignent les essais parus à la fin des années 1970 et au début de la décennie suivante. Qu'il soit question de livres ou de périodiques d'artistes, de mail art ou de pages insérées dans des magazines, force est de constater la récurrence de cette idée d'alternative space ${ }^{3}$.

Évoquant plus particulièrement les périodiques d'artistes, Edit DeAk et Walter Robinson, dans leur contribution à l'ouvrage The New Arts Space, publié en 1978, ont bien souligné les principaux enjeux de telles publications: "Ces magazines [...] incarnent quatre aspects de la raison [d'être] du périodique alternatif: le magazine en tant qu'espace d'exposition, le magazine comme lieu d'une sensibilité communautaire, le magazine indépendant du système commercial des galeries, et le contrôle par les artistes de leur propre parution dans la presse $\mathrm{e}^{4}$. ". On pourrait ajouter à ces quatre points, bien que cela relève de l'évidence, l'abolition de l'unicité de l'œuvre - et par voie de conséquence, d'une certaine préciosité. Par son caractère reproductible, l'utilisation du support imprimé montre en effet par principe l'engagement des artistes en faveur d'un mode de production et de distribution repensé. Parlant du livre d'artiste, l'éditeur et artiste belge Guy Schraenen évoque (rétrospectivement) la révolution qu'il amène sur le plan de la diffusion: "Un mois après sa création, il pouvait y avoir dans plusieurs pays une centaine de personnes en possession de ce livre. L'artiste pouvait ainsi court-circuiter tout un système officiel contraignant. L'œuvre était ainsi mise en circulation dans la foulée de la création ${ }^{5}$ ". Autre acteur incontournable de la publication d'artiste, Ulises Carrion - artiste mexicain émigré aux Pays-Bas insiste sur le fait que, par le support imprimé, les limites spatiales se voient considérablement repoussées: "Un artiste n'a pas besoin de vivre dans une "capitale artistique" pour faire entendre sa voix [...]. L'ubiquité de l'œuvre cesse d'être une caractéristique secondaire et devient essentielle ${ }^{6} \|$.

Ainsi, outre des facteurs techniques qui permettent à l'artiste de gagner en autonomie, le contournement de cette longue suite d'intermédiaires évoquée précédemment a pour conséquence immédiate une accélération de la diffusion des œuvres. Ces dernières n'ont plus à franchir les échelons "labellisants " imposés par la société mais accèdent plus directement à leur but, en l'occurrence le « public » (même si ce public, entendu de manière générique, est
3 Les titres de ces nombreux articles et chroniques en témoignent: «Alternative Space: Artists' Periodicals » (Howardena Pindell, The Print Collectors Newsletter, September/October 1977) ; « The Artist's Book as an Alternative Space » (Kate Linker, Studio International, January 1980) ; «The Page as an Alternative Space " (Marjorie Welish, Art in America, November 1981); "The Page as Alternative Space, 1950 to 1969 " (Barbara Moore et John Hendricks, Artists' Books: a Critical Anthology and Source Book, New York, éd. J. Lyons, 1987).

${ }^{4}$ Edit DeAk et Walter Robinson, "Alternative Periodicals », The New Arts Space: A Summary of Alternative Visual Arts, Los Angeles, ed. B. Reak-Johnson/ Los Angeles Institute of Contemporary Art, 1978, p. 38-39 (trad. de l'auteur).

5 Guy Schraenen, entretien avec Hubert Besacier, Guy Schraenen Collectionneur (fragment), Saint-YrieixLa-Perche, Pays-Paysage, 1995, np.

6 Ulises Carrion, « From Bookworks to Mailworks », 1978, p. 12 (cité dans The Artist Publisher, A Survey by Coracle Press, London, Crafts Council Gallery, 1986, p. 5). Nous pouvons remarquer 
que l'« ubiquité » évoquée par Carrion est une notion phare du texte de Walter

Benjamin, « L'CEuvre d'art à l'ère de sa reproductibilité technique » (1936), Essais 2, trad. M. de Gandillac, Paris, Denoël/Gonthier, 1983.

7 Hervé Fischer, op. cit., p. 5 .

8 Anne Mœglin-Delcroix, Esthétique du livre d'artiste, Jean-Michel Place, Paris, 1997, p. 36. L'auteur rappelle à ce propos la déclaration de Luciano Bartoloni : «Mes livres sont conçus dans la perspective d'être envoyés directement (par la poste) à qui je veux ».

9 Michael Crane, «A Definition of Correspondence Art », dans M. Crane et M. Stofflet (sld), Correspondence Art, Source Book for the Network of International Postal Art Activity,

San Francisco, Contemporary Art Press, 1984 , p. 6.

${ }^{10}$ Michael Crane, « The Origin of Correspondence Art ", ibid., p. 83-116. davantage un certain public). Mais il va de soi qu'en refusant cet ensemble de services, les artistes ont dû lui trouver un « substitut». Ces " sub-cultures », telles que les nomme Hervé Fischer, ont en effet comme seule alternative le recours à des moyens de diffusion plus spécifiques, mais aussi plus discrets, empruntant les voies de ce qu'il appelle les "communications marginales " (marginal media $^{7}$ ). Le réseau postal s'impose dès lors comme le meilleur moyen d'échapper à l'hégémonie du monde de l'art "officiel ", d'en augmenter considérablement le nombre de bénéficiaires, tout en permettant cet élargissement sans précédent des frontières évoquées par Carrion. Par ce biais, atteindre rapidement n'importe quel destinataire devient envisageable, quelle que soit la situation géographique et politique du pays dans lequel vit ce dernier.

\section{Mail Art et Correspondence Art: définition(s)}

Avant de s'arrêter sur un ensemble de pratiques habituellement associées au Mail Art ou Correspondence Art, il importe d'insister sur le fait que l'utilisation de la voie postale ne se réduit pas à un phénomène d' "école " ni à un medium spécifique que serait par exemple la lettre. Si, par le principe de l'abonnement, la revue (ou périodique) recourt naturellement à ce système de distribution, il en va de même pour les livres d'artistes qui anticipent bien souvent les problèmes liés à la diffusion: « les livres aussi circulent par voie postale et leur format est souvent déterminé en conséquence » rappelle Anne Mœglin-Delcroix ${ }^{8}$.

Dans sa définition de "l'art par correspondance » Michael Crane insiste sur le fait que les pratiques réunies sous ce terme ne constituent en aucun cas un mouvement, tout comme elles ne renvoient à aucun « isme ». Selon lui, « la traditionnelle classification historique sous forme de genres, d'écoles et de styles ne peut pas [lui] être appliquée ", justement parce que "l'activité elle-même est une arène plurielle et diverse qui s'est développée au travers de nombreuses attitudes et catégories, et ne peut pas être "épinglée" si facilement par une classification" $»$. Dans un autre texte, Crane dresse d'ailleurs une liste impressionnante de mouvements ou groupes d'artistes ayant utilisé ce mode de diffusion: il y évoque ainsi, parmi d'autres, les Nouveaux Réalistes, l'art conceptuel, mais aussi Fluxus, le Pop Art ou encore l'Arte Povera ${ }^{10}$.

De nombreux mythes se sont construits autour du mail art et certains poncifs ont été considérés comme des éléments suffisants et nécessaires, tel celui du " cachet de la poste faisant foi » comme donnant sens ou crédit à l'œuvre. En réalité, peu d'artistes se 
retrouvent dans cette idée qui ne concerne que certaines démarches auxquelles on ne saurait réduire ce phénomène. Le fait que les spécialistes ou acteurs de ce domaine aient eu du mal à s'accorder ne simplifie d'ailleurs pas la tâche. Évoquant ces pratiques qui utilisent l'institution postale, on rencontrera les appellations « mail art », " correspondence art », " art postal », voire même "Eternal Network ${ }^{11}$ ". Ces différents termes ne sont pas à comprendre comme de simples variations de traductions, mais témoignent d'un certain nombre de dissensions. Ainsi, dans Eternal Network, A Mail Art Anthology ${ }^{12}$, Chuck Welch prétend dans son introduction que l'on peut utiliser indifféremment l'un ou l'autre terme, tandis que Ken Friedman s'applique plus loin à expliquer leur dissemblance. De son côté, le magazine Umbrella (magazine américain dédié à ces pratiques) a publié en 1983 un article intitulé « Mail Art is not Correspondence Art ». L'auteur Robert Morgan y explique que le premier renvoie à « un geste d'exécution faisant référence à une situation d'actualité », alors que le second est plus proche d'un " geste littéraire », moins abstrait et dirigé vers l'intimité du récepteur. Pourtant, dans le numéro suivant, Jack Cracker rétorque - en tant que « mail artiste actif »-que Morgan est bien mal placé pour décider ce qui est ou n'est pas du « Mail Art » et il conteste la différence établie ${ }^{13}$. On remarquera encore que les premiers théoriciens à s'être vraiment intéressés à cette pratique au début des années 1970 que sont Jean-Marc Poinsot ${ }^{14}$ et Hervé Fischer, n'emploient pas la même terminologie bien qu'ils semblent s'accorder sur le fond: « mail art » pour le premier et « art postal " pour le second. Tentant de clore ce débat terminologique dans un article de 1991, Chuck Welch en arrive à la conclusion suivante: "Si la vision d'un "Eternal Network" est vraie, le "correspondence art" et le mail art sont simplement des antécédents historiques des mondes correspondants d'un art en réseau'15 $"$.

Ces désaccords autour de la dénomination adéquate peuvent paraître assez anecdotiques; ils traduisent néanmoins le malaise qui entoure certains aspects de ce phénomène et les pratiques auxquelles on a trop souvent voulu le réduire. Et il est vrai que le principe de l' « art postal » a parfois été « récupéré » au détriment des véritables enjeux - souvent liés à un engagement fort - qui ont conduit les artistes à emprunter cette voie, notamment par l'institution postale elle-même qui souhaite récupérer ce phénomène qu'elle n'a pourtant pas toujours facilité (on se référera à de nombreux catalogues publiés par le Musée de La Poste qui s'arrêtent souvent sur un aspect « décoratif »). Guy Schraenen témoigne en ce sens: « J'ai organisé en 1981 une des plus grandes expositions
11 L'Eternal Network est un concept développé par Filliou qui correspond en français à la notion de « Fête Permanente ».

12 Considéré comme un ouvrage de référence pour le mail art: Eternal Network, A Mail Art Anthology, Toronto, University of Calgary Press, 1994.

13 Robert C. Morgan, «Mail Art is not Correspondence Art », Umbrella, $\mathrm{n}^{\circ}$ 3, vol. 6, 1983 , p. $117-119$ et Jack K. Cracker, «Mail Art Exhibitions \& Competitions : It Ain't (Necessarily) So, Mr. Morgan ", n० 2-5, vol. 6,1984 , p. 85 .

${ }^{14}$ Jean-Marc Poinsot, Mail Art. Communication à distance. Concept., Paris, éd. Cédic, 1971, p. 20 et 28 où l'auteur justifie ce choix.

${ }^{15}$ Chuck Welch, «Mail Art Network: Source and Flow ", Real Life Magazine, 1991, p. 52 . 
${ }^{16}$ Guy Schraenen, op. cit., np. Cette " dégénérescence » est « constatée » par de nombreux auteurs. Voir par exemple Anna Banana, "Mail Art Canada », Correspondence Art, op. cit., p. 254 ; Géza Perneczky, The Magazine Network, Cologne, Soft Geometry, 1993, p. 58.

17 Jean-Marc Poinsot, op. cit., p. 22.

$\mathbf{1 8}$ Hervé Fischer, Art et communication marginale, op. cit. ; voir également Timbres et tampons d'artistes, catalogue d'exposition par Rainer Mason, Genève, Musée d'Art et d'Histoire, Cabinet des Estampes, 1976 ou encore L'Art du tampon, catalogue d'exposition par S. Nagiscarde, Musée de la Poste, 1995.

19 « Correspondence between Brian Lane and Simon Cutts ", The Artist Publisher, op. cit., p. 5 . d'art postal qui aient jamais eu lieu sur les années soixante-dix. Je me suis complètement désintéressé de ce phénomène parce qu'il a été exploité et détourné par des gens inintéressants. Au départ l'idée était intéressante, mais à mon avis, à partir des années 80 elle a complètement dégénéré ${ }^{16}$ ».

Ainsi, il importe de bien garder à l'esprit que le recours au service postal n'est pas toujours motivé par des objectifs similaires: parfois relevant de la simple commodité, il peut être lié à une véritable réflexion sur la diffusion de l'information, sur la mise en circulation de l'œuvre et par conséquent sur les modalités de sa réception. Ce sont ces dernières pratiques qui vont nous intéresser tout particulièrement, et notamment la capacité d'adaptation dont ont dû faire preuve les artistes vis-à-vis de l'institution postale afin de rester cohérents.

\section{L'utilisation de l'institution postale et ses paradoxes}

Il faut dès lors revenir sur un point: alors que la volonté d'échapper aux contraintes inhérentes à un service de diffusion trop lourd et trop réglementé est manifeste, les artistes ont dû, pour s'en affranchir, se confronter à un autre service ayant lui aussi ses règles et usages. La situation peut paraître paradoxale car, comme l'a très justement noté Jean-Marc Poinsot, « si un artiste utilise à ses propres fins l'institution postale, il est obligé de prendre en ligne de compte toutes les contraintes et lois du système qu'il utilise même s'il les tourne en dérision. Placé entre la volonté de prendre possession du pouvoir d'information et celle de contester la forme même du moyen employé, l'artiste devra assumer cette contradiction. [...] L'artiste agit dans un système qui est contraignant au départ et hautement représentatif des lois de notre civilisation ${ }^{17}$ ". Certains artistes résolvent délibérément la contradiction inhérente à ce " changement de service » en jouant avec les signes intrinsèques de l'institution postale ainsi qu'avec les caractéristiques formelles des administrations, utilisatrices massives de ce service. Ainsi, se développent par exemple des pratiques mettant en jeu des timbres et tampons d'artiste ${ }^{\mathbf{1 8}}$. Le mail art est d'ailleurs défini par Simon Cutts et Brian Lane comme " un moyen d'utiliser les rouages administratifs de l' “Establishment” pour une activité subversi$v^{19}$ ". Le plaisir de retourner la " machine " contre elle-même en exacerbant ses codes accentue cette contradiction pour finalement en faire un vocabulaire plastique spécifique. En somme, le fait de passer par le service postal, même s’il sert simplement au départ d'alternative, a pu avoir des répercussions sur la forme même des 

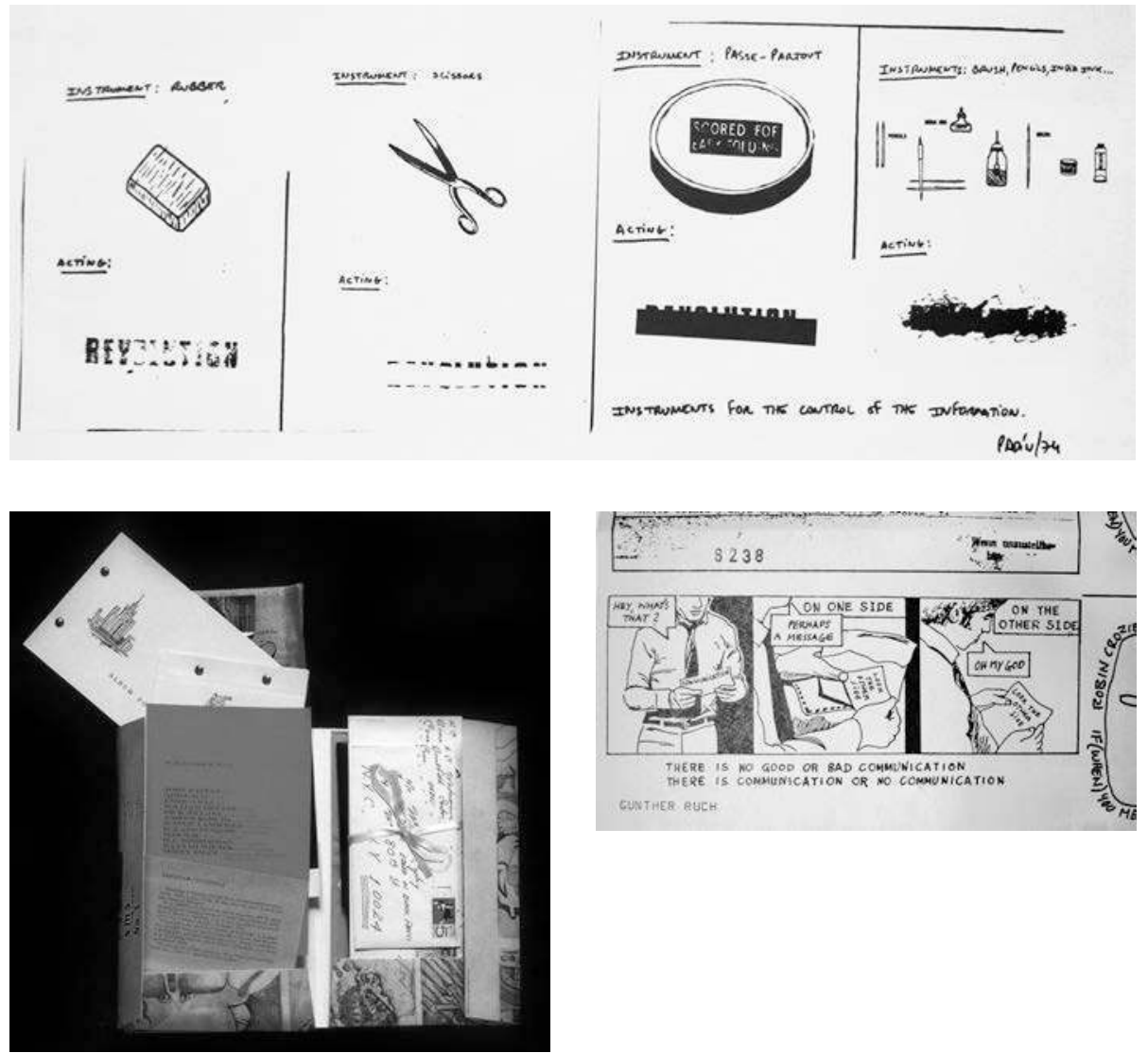

\section{En haut}

Clemente Padin, Instruments for Control of Information, Fandangos, $\mathrm{n}^{\circ}$, automne/hiver 1974, p. 8.

\section{À gauche}

Eric Watier, Papiers,

$\mathrm{n}^{\circ} 2$, octobre 2007.

\section{À droite}

Gunther Ruch,

There is no Good or Bad

Communication, Libellus,

$\mathrm{n}^{\circ} 3$, Anvers, Guy

Schraenen (éditeur), 1980. 

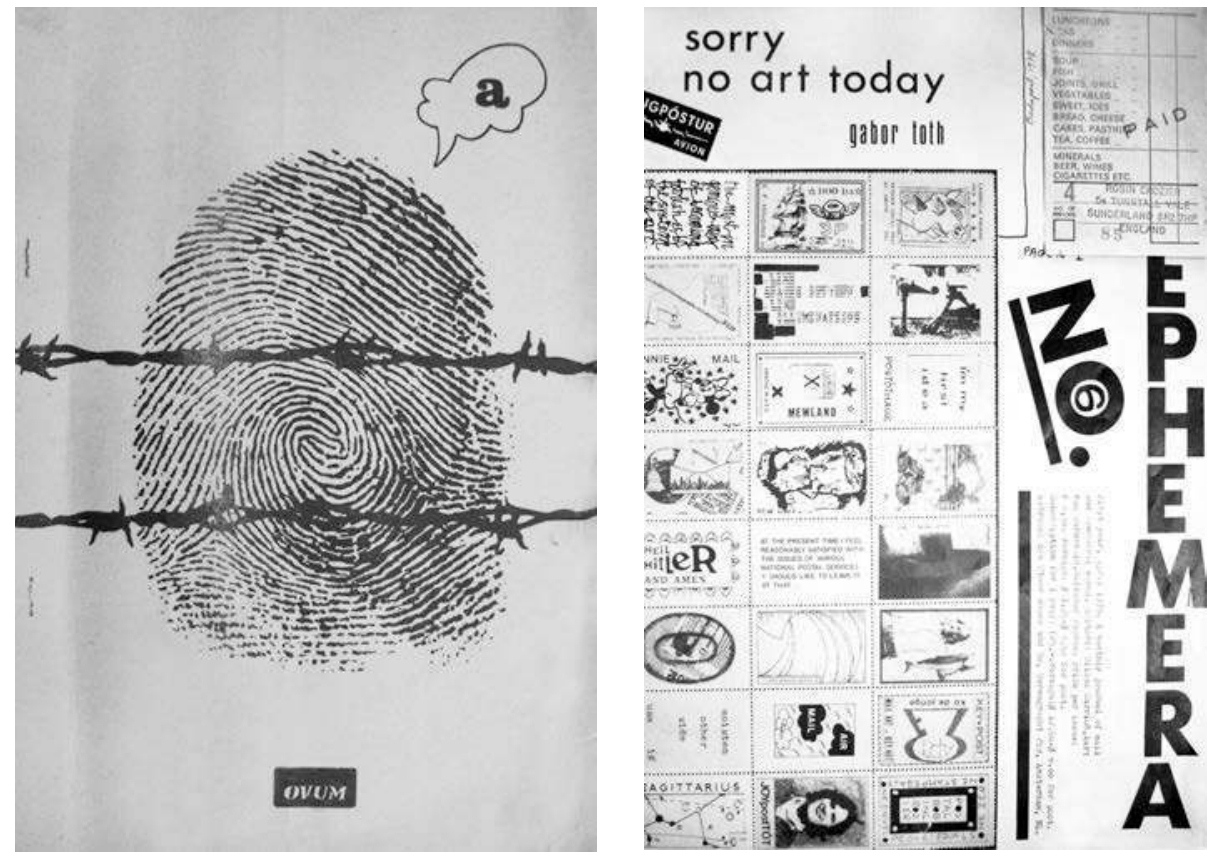

À gauche

Couverture de Ovum, $n^{\circ} 1,2^{e}$ série, Montevideo, Clemente Padin (éditeur), octobre 1973.
À droite

Couverture de la revue

Ephemera, $\mathrm{n}^{\circ} 6$,

Amsterdam,

Ulises Carrion (éditeur), avril 1978. 
œuvres qui, pour certaines, se sont mises à réfléchir (à) cette esthétique de la communication.

Une autre différence essentielle, qui n'a pas encore été évoquée, émane de ces pratiques d'un art par correspondance: s'opposant par essence à la logique consumériste, ces dernières rétablissent l'échange et en revoient les modalités, notamment en remettant au goût du jour les principes du don et de la gratuité. Ce faisant, elles se proposent de réévaluer les rapports entre destinateur et destinataire, allant parfois jusqu'à inverser la « donne ». Car repenser l'échange, c'est aussi revenir sur celui instauré par les mass media et dénoncé par Jean Baudrillard comme étant une " parole sans réponse »: « ce qui caractérise les media de masse, c'est [...] qu'ils fabriquent de la non-communication - si on accepte de définir la communication comme un échange, comme l'espace réciproque d'une parole et d'une réponse, donc d'une responsabilitée ${ }^{20}$ ". L'échange de toute évidence - à partir du moment où il fonctionne à sens unique - n'en est plus véritablement un: ce glissement de " sens ", voire même ce retournement de la notion d' " échange " est au cœur de la réflexion des artistes.

Notons encore que l'utilisation du service postal ne coïncide plus tout à fait, dans l'usage qu'en font les artistes, avec son but initial : la plupart du temps, il ne s'agit plus pour eux de transmettre des informations « utiles » mais bien davantage d'établir des contacts, de construire un réseau, une communauté (" lieu d'une sensibilité communautaire » disent DeAk et Robinson). S'instaure donc vis-àvis de ce service, plutôt qu'un franc détournement, un rapport tactique, par lequel les modalités de mise en circulation ne changent pas à proprement parler. Il est question de subvertir subrepticement le service, non en le transformant ou en le rejetant mais en l'utilisant à des fins et en fonction de références étrangères à ce système ou encore en trouvant la marge de manœuvre laissée par l'institution avec laquelle il faut « composer ${ }^{21}$.

\section{Le rôle de la poste. Un simple moyen?}

Il importe d'insister sur le fait que pour ces artistes impliqués dans une réflexion sur la " communication à distance » ou l' " esthétique de la communication ${ }^{22}$ ", le passage par la poste n'a jamais été la seule alternative; il s'est imposé dans les pratiques avant tout parce que son réseau s'est trouvé être le plus maniable et le plus accessible à l'époque où les artistes ont commencé à s'interroger sur la pertinence des modalités établies de distribution des œuvres d'art. Il n'est en effet pas exclusif et le téléphone comme le
${ }^{20}$ Jean Baudrillard, Pour une critique de l'économie politique du signe, Paris, Gallimard, 1972, p. 208 (cité par Hervé Fischer, op. cit., p. 5).

${ }^{\mathbf{2 1}}$ Hervé Fischer, op. cit., p. 12 : «L'utilisation de la poste, $[. .$.$] implique$ une liaison avec le rythme de la vie quotidienne [...] dans un but un peu détourné, clandestin, a-normal de l'institution postale ».

22 Jean-Marc Poinsot, op. cit. 
23 Hervé Fischer, op. cit., p. 12.

24 La revue évolue d'ailleurs au fil des livraisons et finit par privilégier cet aspect artistique, au détriment des informations sur le Network.

${ }^{25}$ AA Bronson, « L'Humiliation du bureaucrate : les centres alternatifs Musées par des artistes ", Museums By Artists, Toronto, Art Metropole, 1983, p. 30.

26 ibid., p. 34-35. fax ont été utilisés quasi-simultanément (même si on peut émettre l'hypothèse que la «matérialisation » inhérente à la voie postale a pu convenir davantage à certains artistes. Les autres réseaux ont d'ailleurs été plus largement utilisés dans le cadre de recherches proches de l'art conceptuel).

Néanmoins, ce moyen a trouvé son propre langage et le fonctionnement de ce service a insufflé certaines de ses particularités aux œuvres qui l'ont traversé. En outre, la poste a joué selon Fischer le rôle d'une "véritable structure active ", un "rôle d'intégration, compensateur de la marginalité de ces artistes ${ }^{23} »$. On peut d'ailleurs rappeler qu'autour du mail art, ont vu le jour de nombreuses publications périodiques au statut ambigu. Parfois simples " bulletins d'informations ", édités pour donner une visibilité à un réseau donné, ces périodiques ont aussi été un lieu propice au développement de véritables projets artistiques. Dans le réseau proche de Fluxus, la Something Else Press Newsletter de Dick Higgins servait, comme son titre l'indique, à transmettre des informations, de la manière la plus directe possible. Dans ce cas l'utilisation de la poste n'est plus tactique mais revient à sa fonction première. La revue $c c V$ TRE créée par George Brecht a en revanche un statut plus équivoque. Bien qu'organisée autour des événements Fluxus, qu'elle se fait un devoir d'annoncer, cette publication laisse aussi la place à de véritables recherches artistiques prenant en compte tant le support imprimé sur et par lequel elles s'incarnent que la diffusion spécifique qui est celle du périodique. Se développant dans un autre " cercle ", on pourra également évoquer la revue FILE Megazine de General Idea (1971-1989), à la fois lieu de « rassemblement »-par les listes d'artistes appartenant au réseau qu'elle diffuse, les annonces d'évènements qu'elle relaie - et véritable projet artistique en $\mathrm{soi}^{24}$. Le témoignage d'AA Bronson membre fondateur de General Idea - est à ce titre éclairant. En tant qu'artiste canadien, il évoque la situation particulière de son pays, nourri « par la stimulation agressive des revues américaines, de la radio américaine et de la télévision américaine. [...] Nous avions oublié que nous étions nous-mêmes de véritables artistes parce que nous n'avions pas notre place dans les media ${ }^{25}$ ॥. II rappelle à quel point « les revues dirigées par des artistes ont constitué le tissu conjonctif qui nous a permis de découvrir notre existence, ainsi que l'existence d'un véritable milieu de l'art. [...] C'était à l'époque la seule façon de nous voir nous-mêmes, de nous découvrir. [...] Cela ne signifie pas que FILE était une forme de communication pour les artistes; c'était plutôt un moyen de nous voir comme faisant partie de ce réseau de personnalités, comme faisant 
partie d'un “milieu" ${ }^{26}$ ». Ce témoignage montre également l'ambivalence de la position des artistes ayant choisi de se faire entendre via le Network: on voit ici de manière évidente que la frontière entre une situation "subie »- à cause de l'omniprésence des médias dominants - et fortement engagée, liée à un choix politique revendiqué, n'est pas toujours facile à tracer.

\section{L'Eternal Network, une philosophie}

Il serait ainsi erroné de laisser entendre que les acteurs du mail art n'ont pas su trouver de terrain d'entente: de nombreux artistes se sont en effet retrouvés autour de l'idée d'Eternal Network et de l'ensemble du projet philosophique ("filliousophique ») de Robert Filliou, travaillant à rapprocher l'art et la vie, et se prononçant en faveur d'un " échange insouciant d'informations et d'expériences, ni élèves, ni maîtres, parfaite licence, parfois parler, parfois se taire $^{27}$ ». Lorsqu'il s'agit d'exposer leur production - que ce soit au sein d'une publication ou d'une institution artistique - les participants du Network restent en effet intransigeants sur l'idée que parce qu'il n'y a «ni maîtres, ni élèves » - personne n'est en droit de faire valoir une quelconque légitimité pour juger les envois: tout doit être pris en compte, aucun jury ne peut interveni ${ }^{28}$. Comment aurait-il pu en être autrement? Il demeure fondamental pour ces artistes de ne pas retomber dans le travers de ce à quoi ils veulent échapper, tout comme il s'agit de rétablir une relation " horizontale ", abolissant non seulement toute hiérarchie mais aussi tout centre névralgique, toute coordination ${ }^{29}$.

Toutes les idées, convictions, tentatives de repenser un fonctionnement de l'art ressenti comme suranné semblent trouver une synthèse possible dans la pratique de l' "Assembling» (pratique exemplaire par sa position « entre catégories »). L'Omnibus News est le premier magazine du genre et l'initiative en revient à trois artistes munichois, Thomas Niggl, Christian d'Orville et Heimrad Prem. En 1969, ces derniers font circuler un appel à contribution invitant qui le souhaite à participer à une publication : dans l'annonce qu'ils diffusent largement, ils s'engagent - conformément à l'esprit du Network - à ne faire aucune sélection. Toutes les propositions seront en effet publiées, sous réserve que les auteurs fassent parvenir aux éditeurs mille cinq cents copies de leur contribution (les frais de reproduction et d'envois sont donc à la charge des artistes souhaitant collaborer au projet). En contrepartie, une fois les volumes assemblés, chaque participant se verra retourner dix exemplaires de la publication obtenue. Entre mail art et publication d'artiste,
${ }^{27}$ Robert Filliou, La Fête permanente présente (The Eternal Network Presents; Das immerwährende Ereignis zeigt), 1984, p. 116. On trouve ce programme, cet ensemble de «non-règles » inscrit sur l'en-tête du papier à lettre de la Non-École mise en place à Villefranche-sur-Mer et reproduit dans le catalogue.

${ }^{28}$ Comme pour le projet de la Société des Artistes Indépendants de 1917 , ces clauses ne seront pas toujours respectées, ce qui provoquera une vive protestation de la part des artistes. Voir à ce sujet la polémique entourant l'exposition « Mail Art Then and Now » de Franklin Furnace (New York) en 1984 (commissaire: R. Cohen) : cette dernière aura pour conséquence de mettre un terme à la grande vague d'expositions sur le mail art qui déferlait aux États-Unis depuis la fin des années 1970. (Les éléments de cette polémique sont rapportés notamment dans John Held J.-R., «The Mail Art Exhibition: Personal Worlds to Cultural Strategies ", At a Distance, op. cit., p. 89-114.)

29 Si des écrits de type « manifestes » ont pu être énoncés (dans le magazine polonais NET dès 1972, ou par les mail artistes californiens Lon Spiegelman et Mario Lara en 1980), ils ne 
faisaient que formuler une sorte de consensus sous-jacent à cette pratique. On pourra encore remarquer que bien que considéré comme " père du mail art »-Ray Johnson et son école ne correspondent pas tout à fait à la logique de l'Eternal Network (en tant qu'organisée autour d'un pivot, Johnson s'assurant toujours une position de « relais »).

$3^{0}$ Dans la mesure où il n'y a pas de jury, pas de choix à faire, le rôle de l'éditeur se réduit à l'ajout d'une couverture (celle, choisie pour Omnibus, manifeste d'un certain humour (noir), représente un crash entre un bus et un train) et, éventuellement, d'un éditorial et/ou de la liste d'adresse des contributeurs en fin de volume (l'ordre alphabétique a été retenu ici afin de respecter une logique dénuée de toute subjectivité). Comme le note Christian d'Orville dans l'éditorial, le rôle d'éditeur est " rendu » aux artistes, qui peuvent dès lors assumer seuls cette tâche.

\footnotetext{
31 «Assembling ", le terme resté pour désigner ce procédé est d'ailleurs le nom d'une autre publication ultérieure à Omnibus. Sur ce projet, voir Richard Kostelanetz, Assembling, Assembling, (New York, Assembling Press, 1978). En France,
}

l'aventure Omnibus News aboutira à un épais volume de deux cents pages environ, regroupant cent dix-sept artistes de huit pays différents (les contributeurs restent cependant en grande majorité allemands, peut-être pour des problèmes de diffusion de l'appel à contribution).

Avec un tel projet, on voit donc se manifester toutes les ambitions des « mails artistes ": abolition de la différence créateur/lecteur (les créateurs sont aussi les lecteurs puisqu'ils sont les principaux destinataires de ces ouvrages) et revendication de l'appartenance à un réseau. L'information elle-même est disséminée par ce biais et, c'est bien la marque de leur participation à un projet collectif qui est recherchée par les artistes. Les «éditeurs » ne remplissent d'ailleurs plus tout à fait ce rôle - de sélection, d'organisation, de décisions - et ne sont dès lors plus que de simples «compilateurs $^{30}$ ». En outre, un véritable échange est mis en place puisque « contre » leur envoi les artistes reçoivent l'ensemble des contributions et chacun peut à son tour faire circuler les exemplaires. « Une chose reste sûre - lit-on dans la préface - l'aspect expérimental de Omnibus News devrait, à travers l'élimination consciente des valeurs conventionnelles et l'élimination d'orientations thématiques, trouver sa place dans l'opposition au monde officiel de l'art ». Et en effet, si Omnibus News n'a pas eu d'autre livraison, il a impulsé un grand nombre d'initiatives reprenant ce principe, et dans de nombreux pays (États-Unis, Canada, Argentine, Uruguay, Brésil, France, etc.) ${ }^{31}$. Accessibles à tous - sur le plan pratique, par la voie postale, mais également sur le plan économique dans la mesure où, même avec un budget très restreint, n'importe qui peut être à l'origine d'un tel ouvrage - les publications de ce type sont dès lors devenues de véritables plateformes d'échanges; leur fonctionnement a été considérablement facilité par un élargissement des contacts sur le plan international. Non seulement n'importe qui peut lancer une publication, mais n'importe qui - sous réserve qu'il ait accès à l'information, au réseau - peut aussi y participer : si les artistes sont tenus de prendre en charge la reproduction de leur travail, il leur est cependant possible d'adapter leur projet à leur budget. La variété des papiers comme des types ou qualités d'impression que l'on peut trouver en feuilletant Omnibus illustre bien le panel de possibilités qui s'offre aux artistes.

D'autres revues essayeront de rejouer à leur manière cette idée " communautaire ", en modifiant à chaque fois quelques données : c'est le cas de Commonpress (1977-1990) lancé par l'artiste polonais Pawel Pestaz. Ce magazine, bien que souvent considéré comme un assembling, ne demande pas aux contributeurs de faire 
eux-mêmes les copies et pousse plus loin la réflexion sur l'interchangeabilité des rôles. L'idée d'une implication essentielle des artistes participant au projet tant dans la prise en charge matérielle que dans la diffusion de leur travail est malgré tout conservée; le principe de Commonpress est en effet que chaque participant devient tour à tour « éditeur » en prenant en charge un numéro. Il doit alors assurer la reproduction des contributions et proposer un thème directeur ${ }^{32}$. Ainsi, à partir du moment où de nouveaux artistes s'ajoutent à chaque numéro, ce système devient en théorie autonome et est, d'une certaine manière, infini.

L'assembling apparaît donc comme un cas particulier de l'utilisation de la voie postale mais il demeure malgré tout fortement connecté au Network en général. Son fonctionnement - s'il est bien sûr à comprendre par rapport à un contexte donné, tel un instantané - permet d'entrevoir quelques enjeux de l'art par correspondance et la singularité des œuvres par essence collectives qui en découlent. Reste que nombre d'artistes devenus « collaborateurs » ne se sont jamais rencontrés autrement que par le réseau, et grâce au service postal qui relayait leurs échanges ${ }^{33}$.

\section{L'art délivré au service d'une prise de parole}

Extrêmement développées à la fin des années 1970, les publications assembling ont donc largement participé aux nombreux échanges d'œuvres imprimées qui ont revendiqué le droit à un autre point de vue sur l'art que celui imposé par les institutions en place. Mais il ne faut pas oublier qu'au-delà de ces enjeux, ces pratiques ont aussi permis de rétablir le contact avec nombre de pays plus ou moins " coupés » du monde par un régime politique totalitaire, comme par exemple, une partie de l'Amérique Latine et certains pays de l'Europe de l'Est. Le support imprimé, et en particulier le magazine, peut même devenir le lieu d'un engagement politique important pour les artistes, d'une prise de parole quasi «vitale ». Pour eux, comme le note Géza Perneczky, le Network représente " une clé qui donne accès, non pas à la porte de l'art alternatif mais à la porte de l'art contemporain dans son ensemble $34 »$. De plus, si nous avons vu que les artistes en changeant de medium se prononcent contre les travers d'une société seulement tournée vers le profit, il ne faut pas oublier que d'autres se battent dans le même temps contre un régime communiste. Cependant, comme en témoignent les publications - quels que soient leur forme et le nombre d'exemplaires que les artistes sont en mesure de produire -, tous se rejoignent dans les mêmes réseaux et revendiquent le même « droit
Paul Armand Gette fait par exemple partie des artistes à avoir adopté ce principe avec la revue Eter (1966-1967).

32 Le numéro 5 par exemple est pris en charge par Carrion et les contributions rassemblées s'articulent autour du thème de la Boxe. Nous pouvons remarquer que ce fonctionnement revient un peu sur les propos de Christian d'Orville qui, dans l'éditorial d'Omnibus évoqué plus haut, semble considérer que le fait de donner une « orientation thématique » relève d'une tradition appartenant au monde de l'art officiel.

33 Le mail artist anglais Robin Crozier a d'ailleurs mis en exergue cet aspect en proposant à l'occasion d'un projet à ces correspondants de réaliser, sans ne l'avoir jamais vu, son portrait.

34 Géza Perneczky, op. cit., p. 56-57. L'auteur parle même d'un « political mail art». 
35 La deuxième série de Ovum dont il est ici question, publiée de 1973 à 1977 , fonctionne sur le mode de l'assembling (ce qui n'était pas le cas pour la première série). à la parole ». Notons que cet engagement n'est pas sans risque malgré le passage par des voies non-officielles: on pourra rappeler pour preuve l'exemple de Clemente Padin qui, après la parution de cinq numéros de sa revue Ovum ${ }^{35}$ - et malgré la mise en œuvre de " ruses » pour passer au travers des mailles des contrôles - s'est fait arrêter par les autorités uruguayennes et ne fût relâché qu'à la fin de l'année 1979, accusé d'avoir exercé une « activité qui blesse la morale et la réputation de l'armée ".

Qu'il soit question de livres d'artistes, de revues ou de " courriers » au sens large, il reste néanmoins extrêmement difficile de résumer ou même de cerner un phénomène si invisible, par essence disséminé et par principe voué à la prolifération. On peut quand même remarquer que, dans tous les cas, le mot d'ordre est bien de courtcircuiter une institution prédominante. Et il est notable que cette " désintermédiation » s'opère en utilisant les propres armes du système à combattre. Si, comme il est admis dans nos sociétés (et comme cela a été prouvé plus d'une fois), le pouvoir appartient bien à ceux qui détiennent les canaux de l'information, il devient fondamental de s'approprier l'un de ces systèmes et de s'en servir pour disséminer ses propres idées.

\section{Livraison à domicile}

On ne saurait donc trop insister: l'utilisation de la poste n'est pas l'objet principal de ces pratiques. En ce qui concerne l' "Eternal Network », nous avons évoqué le fait que dès que quelqu'un « met un pied » dans le réseau - et s'il veut être au fait de l'activité foisonnante qui s'y développe, il n'a pas d'autre choix - il est aussitôt mis au même niveau que tous les participants, et comme eux, il peut ou doit devenir acteur et non plus simple contemplateur (parfois un peu malgré lui).

L'utilisation de la poste amène encore une autre conséquence qui a jusqu'ici été peu développée. Si le rapport à l'œuvre change dès que celle-ci devient reproductible, ce rapport est également modifié lorsque l'œuvre n'est plus à contempler dans un «temple » de l'art mais à regarder chez soi. Par l'utilisation de la voie postale, l'œuvre est ainsi littéralement livrée. Il ne s'agit pas de sous-entendre que créer un nouveau service - la livraison à domicile - ait pu faire partie des objectifs principaux des artistes, et ce, encore moins dans une visée consumériste qui permettrait à l' " intéressé » de voir (d'avoir) plus, tout en réduisant son investissement: au contraire, beaucoup d'actions ont eu pour conséquence de responsabiliser davantage les spectateurs, comme les artistes. 
Et pourtant, parce que chacun peut avoir accès à une proposition artistique élaborée à des milliers de kilomètres - et éventuellement par des dizaines de personnes différentes -, cet aspect a son importance. Parce qu'elle nous est livrée et parce qu'elle emprunte le support imprimé - l'un et l'autre étant d'ailleurs dans ce cas liés - l'œuvre perd son caractère "sacré » ou « intouchable » mais plus encore, elle modifie la posture du destinataire. Ce dernier se voit forcé d'échanger sa «peau de spectateur » contre celle de « lecteur ». Un rapport beaucoup plus personnel à l'œuvre s'instaure, sans contrainte temporelle: on peut aller à son rythme dans la « consultation » de l'œuvre, et y revenir autant de fois qu'on le souhaitera. Pour le cas particulier de la publication périodique (mais cela peut être également vrai pour des livres) le rapport au temps est encore en jeu dans l'attente/désir de l' " abonné », qui sait qu'il doit recevoir un certain nombre de livraisons. C'est ce qu'exemplifient les Suspense Poems de 1961 de Robert Filliou, l'un des premiers artistes à avoir pris en compte le délai de livraison postal. La lettre qu'il envoie aux potentiels abonnés de ce work in progress en explique le principe: " vous recevrez chaque 2 ou 3 semaines franco de port et d'emballage, un vers-objet, jusqu'à ce que le poème soit terminé (environ 5 envois). Le tout accroché par vos soins dans l'ordre d'arrivée formera un objet poème de Robert Filliou ${ }^{36}$ ».

Dans le même sens, la nature même d'une publication peut être modifiée dès lors que sont prises en compte les modalités de sa réception: certains artistes profitent de ce caractère pour confronter le lecteur à des projets nécessitant pour leur compréhension des manipulations particulières. Déjà présente en raison de la forme même de in-folio, pour lequel il faut tourner les pages, les publications sous forme de boîtes ou d'enveloppes ${ }^{37}$ induisent encore davantage une telle participation du " spectateur »: le périodique S.M.S. (Shit Must Stop) des éditions Letter Edged in Black Press, réalisé autour du peintre William Copley est de celles-là. Cette étonnante et ambitieuse publication prend la forme de portfolios - six ont été réalisés entre février et décembre 1968 - contenant chacun une dizaine de contributions originales, d'artistes aussi divers que Lichtenstein, Duchamp, Kosuth, Cage, Christo, Arman, La Monte Young, pour ne citer que quelques uns des soixante-douze artistes ayant participé au projet. Chaque " pièce " contenue dans la pochette est pour le lecteur l'occasion d'une nouvelle approche, allant du simple « déballage » (comprenant parfois plusieurs étapes de dépliage voire de véritables déploiements) à la fabrication pure et simple de la pièce ${ }^{38}$. Selon Rachel Epp Buller, ces artistes se rejoignent tous autour de Copley par le fait qu'ils « préfèrent que l'on
${ }^{36}$ Lettre écrite par Robert Filliou, reproduite dans La Fête permanente présente, op. cit., p. 130. À la différence des œuvres sur lesquelles met l'accent cette étude, les poèmes en question sont des « pièces uniques» (et chaque " vers-objet » se matérialise sur une petite planche de bois, qui pourra être rattachée aux autres par de simples petits crochets).

${ }^{37}$ Fonctionnant sur ce principe, on pourra citer la petite publication Semina (1955-1964) de Wallace Berman, les « boîtes » Fluxus publiées à partir de 1963 ou encore Aspen, " the Magazine in a box » (1966-1969) édité par Phyllis Johnson.

${ }^{38}$ Ainsi, Arman fournit dans S.M.S. $n^{\circ} 4$ tout le matériel nécessaire et, dans le $n^{\circ} 5$, il incombe à chacun de reconstituer la pièce de Yoko Ono qui « livre» une tasse brisée, allant même jusqu'à fournir le tube de colle qui permettra d'en recoller les morceaux.

39 On pourrait cependant nuancer cette remarque en notant que la plupart des artistes qui prennent part à l'aventure S.M.S. n'ont pas développé au-delà de cette expérience une pratique allant dans ce sens.

Rachel Epp Buller, « Mail Order Art : the S.M.S. Portfolios of 1968 ", Oculus, Vol. 2, $\mathrm{n}^{\circ} 1,1999$, p. 10 
$4^{0}$ Leszek Brogowski, «Manipuler/Lire », article en ligne [page consultée le 23/06/07] (pour les éditions Incertain Sens) : http://www.uhb.fr/alc/g $\mathrm{rac} /$ incertain-sens/ analyse $20 Y \% 20$ Chaudou et\%2omanipuler\%2olire. $\mathrm{htm}$ joue avec leurs œuvres sur la table d'une cuisine plutôt qu'ils soient expédiés derrière une vitrine dans un musée ${ }^{39}$ ». Cette remarque amène une autre considération: si, nous l'avons vu, l'accès au " public » se fait de manière beaucoup plus directe grâce au recours à l'institution postale, il est aussi à remarquer que cette diffusion " sans délai » tente de retarder par ce même biais l'accès pour ces œuvres aux " grandes » collections: « les collections sont en effet les cimetières de l'art, et l'on sait que sans cimetières, pas de culture humaine du tout. C'est là que les œuvres, pourrait-on penser, doivent conclure leurs vies, après avoir longtemps bien servi la société. Mais cette société se débarrasse de l'art en raccourcissant le chemin entre l'atelier et la collection et en privant de plus en plus les artistes d'autres modalités d'exister par l'art » remarque Leszek Brogowski ${ }^{40}$. En rétablissant un contact direct avec le spectateur, les publications préviennent ce risque et, d'une certaine manière, retrouvent leur place au sein de la société.

\section{Résonances actuelles}

Si la fin des années 1960 a vu la mise en place d'un certain nombre de réseaux, qui passent le plus souvent par la voie postale et tentent, pour la plupart, de trouver une alternative aux services intermédiatiques qui se sont construits autour de la commercialisation de l'objet d'art, il s'agit aussi (voire surtout) pour les acteurs de ces réseaux de reprendre une parole qui, soit ne leur a jamais été accordée, soit refuse dorénavant de subir les déformations inhérentes aux « filtres » des médias. La forme même de l'œuvre est reconsidérée et doit s'adapter à ces nouveaux principes de diffusion. Mais, quel que soit le medium emprunté, les modalités de réceptions sont également profondément modifiées et la place du spectateur réévaluée. Comme en écho à ces pratiques, nous pouvons d'ailleurs observer que certains projets contemporains jouent des apories ou des paradoxes de ces systèmes, renouvelant ainsi ou poursuivant les remises en cause et la dimension critique que permet cet art délivré. La prise en compte de la notion de service va ainsi s'accentuer dans nombre de pratiques, faisant écho à son développement dans la société en général. Le principe d'abonnement, bien sûr antérieur à la société de service mais dont le développement sans précédent est pourtant symptomatique de celle-ci, est l'un des points qui reste au cœur des questionnements des artistes, pour lesquels la diffusion de leur travail demeure essentielle. Eric Watier et Pascal Le Coq, tous deux à l'origine de revues d'artistes, abordent chacun cette question à sa manière. 
Le premier a réalisé entre 1996 et 2002 une série de petites revues parmi lesquelles Architectures remarquables (1996-97), Paysages avec retard (1997), Paysage (détails) (2002). Pour les diffuser, l'artiste a mis au point un principe d'abonnement inversé : les abonnés le sont " automatiquement » et à leur insu mais peuvent, s’ils le souhaitent, se désabonner (grâce à un papillon de désabonnement livré avec la publication). Ce faisant, l'artiste retourne véritablement la situation dans la mesure où ce n'est plus le « client » qui passe une convention avec un « fournisseur » pour bénéficier d'un service donné mais l'inverse (on retrouve dans cette stratégie du « pied dans la porte » un écho à la diffusion de publicités, newsletters, que nous subissons plutôt que nous ne les demandons, et contre lesquelles il faut se manifester pour ne plus les recevoir). En outre, il est précisé au nouvel abonné qu'il ne recevra la petite publication que « de temps en temps », qu'elle soit mensuelle (pour les premières revues) ou quotidienne. Si l'on considère que l'abonnement, tel qu'il se définit habituellement, permet de bénéficier d'un service régulier, valable pendant un temps déterminé à l'avance, le fait d'être abonné et pourtant de ne pas recevoir la publication régulièrement est quelque peu déroutant, et s'oppose à la définition même du périodique. Le paradoxe est encore plus grand quand on sait que la plupart des abonnés involontaires ne recevront finalement qu'un seul numéro du périodique.

Pascal Le Coq, dans sa publication « à géométrie variable ${ }^{41}$ » $O X O$, qui paraît depuis 1996, " respecte » quant à lui les engagements qui le lient à ses abonnés. Il va même jusqu'à en assimiler tous les codes, s'emparer de manière excessive de leur "langage »: s'appropriant les systèmes commerciaux liés aux périodiques, il propose ainsi, par exemple, aux lecteurs de parrainer de nouveaux abonnés en échange de cadeaux «publicitaires ». Affichant une transparence " exemplaire »-pour ne pas dire exagérée - il rend compte aux lecteurs de tous les choix qu'il opère, toutes les décisions et les règles qu'il s'oblige à suivre. Dès lors, il rend aussi complice ce dernier de toutes les transgressions ou « trahisons » qu'il réussira à imposer à son propre système.

Si nous avons porté notre attention sur les œuvres imprimées (et tout particulièrement les périodiques), il est indéniable que la plupart des avantages - ou en tout cas modifications - mises en avant par les réseaux « courriers » se retrouvent sur les réseaux électroniques. Des échanges d'une nature qui n'est plus commerciale peuvent de la même manière se mettre en place, tout comme il est devenu possible de concevoir un art détaché de tout centre, un art « multi-pôle » où le rôle de chacun n'est plus tant déterminé par la
${ }^{41}$ Le nombre d'abonnés détermine la forme de la revue (tirage, nombre de pages, technique, noir et blanc ou quadrichromie). Détail du principe de variabilité, voir : $O X O \mathrm{n}^{\circ} 10$, éd. de l'artiste, Pantin, 1999. Le principe de variabilité d'OXO est également reproduit dans la brochure publiée à l'occasion de l'exposition «Critique et Utopie, livres d'artistes », La Criée, Rennes, janvier/février 2001. 
42 Michael Crane, Correspondence Art, op. cit., p. 20.

$43 \mathrm{Cf}$. les actes du colloque Ec/Arts3, Pratiques artistiques et nouvelles technologies, éc/artS/ Ministère des affaires étrangères-adpf, 2002. Voir notamment les participations de Michel Valensi, "Gratuité des savoirs et commerce du livre ", p. 151-155 et Hervé Fischer, « Du livre, du papier et du cathodique ", p. 300-303.

44 «Papiers - est une publication incohérente et apériodique à imprimer chez soi. Vous pouvez vous désabonner en retournant ce mél. Vous pouvez aussi abonner vos amis. ". Papiers est diffusé depuis septembre 2007. renommée et/ou la situation géographique. Peut-être même, l'œuvre diffusée par Internet permet-elle une interaction encore plus grande que celle des exemples précédemment évoqués. Similairement, parce que sa réception se fait, sinon à domicile, du moins dans un rapport privé qui n'est pas celui du musée, le spectateur se retrouve dans une position de lecteur non assujetti à la contrainte temporelle. Mais le réseau Internet, par l'ensemble de possibilités qu'il amène, est aussi l'occasion d'envisager de nouveaux rapports à l'œuvre qui lui sont propres, et que prennent en compte les innombrables projets d'artistes (y compris les revues d'artistes en ligne) qui naissent chaque jour. Déjà en 1984, Michael Crane notait que « comparé aux nouveaux moyens électroniques tels que les réseaux informatiques privés et les réseaux interactifs des télécommunications, l'utilisation du courrier est lente et nostalgique ${ }^{42}$ " (qu'en dirait-il aujourd'hui!). Il serait cependant erroné d'en tirer la conclusion que l'un puisse se substituer à l'autre (tout comme il a été montré que, contrairement aux adages qui se sont multipliés à la fin du siècle dernier, le livre n'est pas près de céder la place au fichier électronique ${ }^{43}$ ). Si certaines publications « papier » ont connu ou connaissent une seconde vie sur le réseau, c'est bien souvent parce que cela permet de réduire des coûts de productions devenus trop lourds à assumer. Mais nous pouvons également souligner que la plupart du temps pour ces dernières, il n'y a pas de véritable prise en compte des possibilités du nouveau support sur lequel elles se « matérialisent » et les « pages » Web qu'elles occupent pourraient tout aussi bien être imprimées. La dernière « publication » d'Eric Watier entre d'ailleurs en résonance directe avec ces questionnements: bien qu'uniquement diffusée par courriel, cette dernière s'intitule significativement Papiers et, comme l'indique le bref message qui accompagne le fichier, elle est « à imprimer chez-soi44 ». L'artiste pointe dès lors l'aspect économique lié à la diffusion par Internet, sans pour autant renoncer à la matérialisation de sa revue, devenue à la charge du destinataire.

Plutôt que de remplacer l'un par l'autre, on peut encore remarquer que nombre d'artistes se servent également d'Internet comme d'un moyen d'information complémentaire sur une publication qui continue d'exister sur papier: c'est le cas par exemple des publications de Céline Duval qui sont relayées au fur et à mesure de leur parution. On trouve de la même manière sur le site de Watier une présentation de ses publications qui, pour certaines, sont d'ailleurs librement téléchargeables. Pascal le Coq a également recours à Internet - par l'intermédiaire d'un blog - afin de compléter les « informations » diffusées dans sa revue: dans ce dernier 
cas, il y a presque une croissance parallèle qui se met en place, deux directions distinctes qui se développent sans jamais se superposer complètement.

La prise en compte des particularités du support et des modes de diffusion demeure essentielle pour la compréhension de ce type de projet qui, contrairement à ce que l'on a parfois sous-entendu ou cru, n'est pas qu'une « idée » mais prend toute sa force et son sens dans sa présentification. Malgré des enjeux communs et un développement quasi simultané45, œuvres imprimées (et diffusées comme telles) et œuvres numériques n'appartiennent pas à la même " réalité » et ne se posent pas dans un rapport de concurrence l'une à l'autre. Pourtant, elles se placent ensemble en faveur d'une circulation en réseaux, où chacun peut avoir son rôle à jouer; elles se disséminent dans un domaine qui échappe - pour un temps au moins - aux circuits traditionnels de l'art.
45 Ce parallèle est bien mis en évidence par Stephen Perkins dans son article «Assembling Magazines and Alternative Artists' Networks ", At a Distance, op. cit., p. 392-407.

\section{Marie Boivent}

